\title{
Genetic Mapping of Tolerance to Iron Deficiency Chlorosis in Peanut (Arachis Hypogaea L.)
}

\section{Ankur D. Tayade}

University of Agricultural Sciences

Babu N. Motagi ( $\sim$ bnmotagi@gmail.com )

University of Agricultural Sciences https://orcid.org/0000-0001-6113-0667

Mangesh P. Jadhav

University of Agricultural Sciences

Anjum S. Nadaf

University of Agricultural Sciences

Rajshekar V. Koti

University of Agricultural Sciences

\section{Sunil S. Gangurde}

International Crops Research Institute for the Semi-Arid Tropics (ICRISAT)

\section{Vinay Sharma}

International Crops Research Institute for the Semi-Arid Tropics (ICRISAT)

\section{Rajeev K. Varshney}

Murdoch University

\section{Manish K. Pandey}

International Crops Research Institute for the Semi-Arid Tropics (ICRISAT)

\section{Ramesh S. Bhat}

University of Agricultural Sciences https://orcid.org/0000-0002-1120-7088

\section{Research Article}

Keywords: Peanut, tolerance to iron deficiency chlorosis (IDC), VCR, SCMR, high-density genetic map, major main effect QTL, candidate gene

Posted Date: January 18th, 2022

DOI: https://doi.org/10.21203/rs.3.rs-1211673/v1

License: (c) (i) This work is licensed under a Creative Commons Attribution 4.0 International License. Read Full License

Version of Record: A version of this preprint was published at Euphytica on March 17th, 2022. See the published version at https://doi.org/10.1007/s10681-022-02996-z. 


\section{Abstract}

Iron deficiency chlorosis (IDC) under calcareous and alkaline soils is a significant abiotic stress affecting the growth and yield of peanut. In this study, the genomic regions governing IDC tolerance were mapped using a recombinant inbred line (RIL) population derived from TMV 2 (susceptible to IDC) and TMV 2-NLM (tolerant to IDC), which was phenotyped during the rainy seasons of 2019 and 2020 in the iron-deficient calcareous plots. The best linear unbiased prediction (BLUP) values for IDC tolerance traits like visual chlorotic rating (VCR), and SPAD chlorophyll meter reading (SCMR) were used for QTL analysis along with a genetic map carrying 700 GBS-derived SNP, AhTE and SSR markers. In total, 11 and 12 maineffect QTLs were identified for VCR and SCMR, respectively. Among them three QTLs were major with the phenotypic variance explained (PVE) of 10.3-34.4\% for VCR, and two QTL were major for SCMR with PVE of 11.5-11.7\%. A region (159.3-178.3 cM) on chromosome Ah13 carrying two QTLs (one each for VCR and SCMR) was consistent with the previous report. A SNP marker, Ah14_138037990 identified from single marker analysis for VCR was located in the intronic region of the gene Arahy.QAOC1, which is important for protein-binding. Overall, this study identified new QTLS and also validated QTL for IDC tolerance. These genomic resources could be useful for genomics-assisted breeding of peanut for IDC tolerance.

\section{Introduction}

Peanut (Arachis hypogaea L.) is a major legume food and oilseed crop mainly grown on sandy, loamy, and clayey soils (Zhao et al. 2015) across the globe. Peanut grown on clay soils suffers from iron deficiency chlorosis resulting in yellowing and stunted growth symptoms and pod yield loss of 16-32\% (Singh 2001). These symptoms are generally related to the important roles of iron in photosynthesis, respiration, nitrogen fixation, hormone production, chlorophyll formation apart from being a component of various redox and iron-sulphur enzymes in plants (Zheng 2010). Acute iron deficiency can lead to plant death and even complete crop failure. Foliar application of salicylic acid and sodium nitroprusside (a nitric oxide donor) could reduce the IDC symptoms and promote iron uptake, translocation and activation (Kong et al. 2014). The treatment also modulated the balance of mineral elements and protected iron deficiency induced oxidative stress.

The mechanisms which reduce the adverse effects of iron deficiency by increasing the soluble iron have been identified (Römheld \& Marschner 1986). Molecular components of iron uptake and transport have also been characterized. In peanut, the full-length cDNA of AhFRO1 was isolated and found to code for Fe(III)-chelate reductase, a membrane protein with role in iron uptake (Ding et al. 2009). Later, AhNRAMP1, coding for a functional iron transporter, was found to be induced by iron deficiency in both roots and leaves. The study also suggested its role in iron acquisition in peanut plants (Xiong et al. 2012).

Recent genomics studies using the RIL population of TAG $24 \times$ ICGV 86031 and a map of 191 SSR markers identified two major QTLs for VCR with the highest PVE of 31.8\% and a single major QTL for SCMR with a PVE of 11.0\% (Pattanashetti et al. 2020). Subsequently, Pandey et al. (2021) improved the genetic map for TAG $24 \times$ ICGV 86031 using 1028 SNP loci genotyped with a high-density 58K "Axiom_Arachis" array and 177 SSR markers. The genome-wide QTL analysis identified a single major main-effect QTL for VCR with a PVE of 33.9\% and two major main-effect QTLs for SCMR with the highest PVE of 22.4\%. These identified QTLs shared a common genomic region on chromosome B03 on diploid genome corresponding to Ah13 of tetraploid genome.

TMV 2 and TMV 2-NLM showed almost similar levels of susceptibility and tolerance to IDC when compared to TAG 24 and ICGV 86031, respectively indicating the suitability of a mapping population derived from them for mapping IDC tolerance. The RIL population derived from TMV 2 and TMV 2-NLM has demonstrated its utility in mapping complex traits (Hake et al. 2017, Jadhav et al. 2021) since the latter parent is an EMS-derived mutant of the former, and helps in subtracting out a major genomic portion common to both the parents. Therefore, an effort was made in this study to map the genomic 
regions governing IDC tolerance using a RIL population derived from TMV 2 and TMV 2-NLM which was phenotyped during the rainy seasons of 2019 and 2020 in the iron-deficient calcareous plots, and a dense genetic map of GBS-derived SNP, AhTE and SSR markers.

\section{Materials And Methods}

\section{Population Development and phenotyping}

A RIL population developed (Pattanashetti 2005) from the cross between TMV 2, an elite variety of peanut and its EMSmutagenized derivative TMV 2-NLM (Narrow Leaf Mutant) (Prasad et al. 1984) was used for mapping the tolerance to iron deficiency chlorosis. TMV 2 is a Spanish bunch cultivar with susceptibility to IDC (Boodi et al. 2016), while TMV 2-NLM (Prasad et al. 1984) is a semi-spreading cultivar with tolerance to IDC. Phenotypic and genotypic differences between TMV 2 and TMV 2-NLM have been previously reported by Hake et al. (2017).

$\mathrm{F}_{19-20}$ generations of the 432 RILs together with the parents were grown during the Rainy-2019 and Rainy-2020 at College of Agriculture, Vijayapur, University of Agricultural Sciences, Dharwad, India. During each season, the RILs were grown in two replications with a spacing of $30 \times 10 \mathrm{~cm}$ with recommended agronomic practices. Soil properties of experimental sites during Rainy-2019 and Rainy-2020 are given in Supplemental Table S1. The climatic factors during the crop period (June to October) at Vijayapur in 2019 and 2020 information given (Supplemental Table S2). Visual chlorotic rating (VCR) at 30, 60 and 90 days after sowing (DAS) was recorded as per the scale proposed by Singh and Chaudhari (1993) based on the severity and the spread of interveinal chlorosis on the leaves where 1 is normal green leaves with no chlorosis, 2 is green leaves but with slight chlorosis on some leaves, 3 is moderate chlorosis on several leaves, 4 is moderate chlorosis on most of the leaves and 5 is severe chlorosis on all the leaves on an overall line basis (Fig. 1a). SPAD chlorophyll meter reading (SCMR) was recorded at 30,60 and 90 DAS using SPAD 502 (Konica Minolta, Japan).

Analysis of variance (ANOVA) was performed for each trait to test for the signi匹cant differences among the RILs. Phenotypic coef $\llbracket$ cient of variation (PCV), genotypic coef $\square$ cient of variation (GCV) and broad sense heritability $\left(h^{2}{ }_{B S}\right)$ were estimated using the plant breeding package Windostat ver. 8.5 (Indostat Services, Hyderabad, India, https://www.indostat.org/agriculture.html). Pearson's correlation coef $\square$ cients $(r)$ among the different traits were estimated over the seasons using the 16th version of SPSS. For further analysis, best linear unbiased prediction (BLUP) values for VCR and SCMR at the three stages (30,60 and 90 DAS) across the seasons were calculated using the Ime4 package of R.

\section{Population genotyping and map construction, QTL and candidate gene discovery}

The genetic map with 700 mapped markers (SNP, AhTE and SSR) previously constructed by Jadhav et al. (2021) was used for QTL mapping. The main-effect QTL analysis was carried out using the "Composite Interval Mapping (CIM)" approach (Zeng 1994) with Model 6 and a scanning distance of $1.0 \mathrm{cM}$ between markers and moving window size of $10.0 \mathrm{cM}$ using Windows QTL Cartographer version 2.5 (Wang et al. 2007). Forward-backward stepwise regression method was used to set the marker cofactors for the background selection. Highest peak was considered to locate QTL where the distance between the peak and the QTL was less than $5.0 \mathrm{cM}$. Permutation $(1,000)$ test was performed to work out the threshold and identify the significant QTL. The QTLs with > 3.0 LOD and phenotypic variance explained (PVE) $>10 \%$ were considered major effect QTLs for a particular trait. Those with a PVE $<10 \%$ were considered as minor effect QTLs. Based on the trait name and chromosome number, the QTLs were named, where the first letter ' $q$ ' indicated the QTL and the abbreviated capital letters indicated the trait followed by the chromosome number and the numerical number indicating the serial number of the QTL for a trait. For instance, qVCR30_Ah04-1 the first QTL for visual chlorotic rating 30 days after sowing was detected on chromosome Ah04. 
Analysis for the epistatic QTL $(\mathrm{Q} \times \mathrm{Q})$ was conducted using the function "two-dimensional scanning ICIM-EPI"

implemented in inclusive composite interval mapping (ICIM) software version 4.1 (Wang et al. 2014) with $5 \mathrm{cM}$ step and 0.001 probability mapping parameters in stepwise regression. The minimum threshold LOD value for significant epistatic QTL was set at 3.0. Multiple QTL Mapping was performed with the r/qtl package's stepwise qtl () function (https://rqtl.org/). Association of the markers with the VCR and SCMR was tested by single marker analysis (SMA) using the $\operatorname{Im}$ () function (linear regression) of R programme. Putative genes were identified for the major QTL clusters and selected markers using the GBrowse https://www.peanutbase.org/ gb2/gbrowse/arahy.Tifrunner.gnm2/) at PeanutBase.

\section{Results}

IDC tolerance-related traits such as VCR and SCMR across the stages (30, 60 and 90 DAS) showed normal distribution (Fig. 2) in the rainy seasons of 2019 and 2020. ANOVA showed significant differences for VCR and SCMR among the RILs (Supplemental Tables 3 and 4). Across the years, TMV 2 showed susceptible response to IDC over all the three stages with VCR ranging from 3.0 to 4.0 and SCMR ranging from 17.1 to 29.9 , while TMV 2-NLM was found to be tolerant to IDC with VCR ranging from 1.0 to 2.0 and SCMR ranging from 28.3 to 40.2 (Table 1). Wide variation was observed among the RILs for VCR, which ranged from 1.0 to 5.0 over the three stages across the years, while SCMR ranged from 2.8-52.9 (Fig. 1b and 1c; Table 1). The average VCR score increased from VCR30 to VCR60, but it decreased in VCR90 with the recovery of some of the RILs. The average SCMR reduced from SCMR30 to SCMR60, but increased in SCMR90 again with some RILS showing recovery (Fig. 2).

Table 1

Estimates of genetic variability for tolerance to iron deficiency chlorosis among the RILs of TMV 2 and TMV 2- NLM of peanut

\begin{tabular}{|c|c|c|c|c|c|c|c|c|c|}
\hline \multirow[t]{2}{*}{ Traits } & \multicolumn{2}{|c|}{ Parents } & \multicolumn{2}{|l|}{ RILs } & \multirow{2}{*}{$\begin{array}{l}\text { PCV } \\
\text { (\%) }\end{array}$} & \multirow{2}{*}{$\begin{array}{l}\text { GCV } \\
(\%)\end{array}$} & \multirow{2}{*}{$\begin{array}{l}h^{2}{ }_{B S} \\
(\%)\end{array}$} & \multirow[t]{2}{*}{ GA } & \multirow{2}{*}{$\begin{array}{l}\text { GAM } \\
(\%)\end{array}$} \\
\hline & TMV 2 & $\begin{array}{l}\text { TMV 2- } \\
\text { NLM }\end{array}$ & Mean & Range & & & & & \\
\hline VCR30 & 3.0 & 1.0 & 1.5 & $1.0-4.0$ & 10.8 & 9.0 & 68.7 & 0.2 & 15.3 \\
\hline VCR60 & 4.0 & 2.0 & 2.0 & $1.0-5.0$ & 16.7 & 16.0 & 91.5 & 0.6 & 31.5 \\
\hline VCR90 & 4.0 & 2.0 & 1.9 & $1.0-5.0$ & 31.1 & 30.8 & 98.0 & 1.2 & 62.8 \\
\hline SCMR30 & 29.9 & 40.2 & 30.5 & $4.0-47.4$ & 11.2 & 8.2 & 52.9 & 3.7 & 12.2 \\
\hline SCMR60 & 17.1 & 28.3 & 21.2 & $2.8-48.8$ & 17.6 & 12.7 & 51.9 & 4.0 & 18.8 \\
\hline SCMR90 & 20.1 & 32.3 & 28.4 & $2.8-52.9$ & 18.2 & 16.3 & 80.2 & 8.5 & 30.0 \\
\hline NPPP & 7.8 & 3.1 & 6.1 & $1.3-17.4$ & 29.7 & 26.6 & 80.4 & 3.0 & 49.2 \\
\hline PYPP & 5.6 & 2.4 & 4.0 & $0.4-15.1$ & 28.1 & 23.4 & 69.2 & 1.6 & 40.0 \\
\hline
\end{tabular}

Phenotypic coefficient of variation (PCV) and genotypic coefficient of variation (GCV) for VCR and SCMR increased over the stages. Highest PCV and GCV observed for VCR were $31.1 \%$ and $30.8 \%$, respectively. SCMR showed the highest PCV and GCV of $18.2 \%$ and $16.3 \%$, respectively. Heritability and genetic advance over mean (GAM) also increased over the stages for VCR and SCMR. The highest heritability and GAM recorded for VCR were $98.0 \%$ and $62.8 \%$, respectively, while SCMR showed the highest heritability and GAM of $80.2 \%$ and $30.0 \%$, respectively. 
VCR across the stages showed positively significant correlations (0.3-0.7). SCMR also showed positively significant correlations (0.3-0.5). However, VCR and SCMR were significantly negatively correlated across all the three stages (-0.2 to -0.8) (Table 2). VCR across the stages showed a significantly negative correlation with the number of pods per plant (NPPP) and pod yield per plant (PYPP), while SCMR showed significantly positive correlations with productivity traits. Multiple regression analysis showed the significant coefficients for VCR60 and SCMR30 with the model showing a multiple $\mathrm{R}^{2}$ of $16.2 \%$ ( $\mathrm{F}$ of 10.1 with $\mathrm{p}$ value $<0.05$ ).

Table 2

Pearson correlation coefficients for iron deficiency chlorosis related traits among the RILs of TMV 2 and TMV 2-NLM of peanut

\begin{tabular}{|c|c|c|c|c|c|c|}
\hline Traits & VCR30 & VCR60 & VCR90 & SCMR30 & SCMR60 & SCMR90 \\
\hline VCR30 & 1.0 & & & & & \\
\hline VCR60 & $0.3^{\star \star}$ & 1.0 & & & & \\
\hline VCR90 & $0.3^{\star \star}$ & 0.7 ** & 1.0 & & & \\
\hline SCMR30 & $-0.5^{\star \star}$ & $-0.3^{\star \star}$ & $-0.2^{\star \star}$ & 1.0 & & \\
\hline SCMR60 & $-0.3^{\star *}$ & $-0.8 * \star$ & -0.6 ** & $0.3^{\star \star}$ & 1.0 & \\
\hline SCMR90 & $-0.3^{\star \star}$ & $-0.6 * \star$ & $-0.8^{\star \star}$ & $0.3^{\star \star}$ & $0.5^{\star \star}$ & 1.0 \\
\hline NPPP & -0.1 & $-0.3^{\star \star}$ & $-0.2^{\star \star}$ & $0.2^{* *}$ & $0.2^{* *}$ & 0.1 ** \\
\hline PYPP & -0.0 & $-0.3^{\star \star}$ & $-0.2^{\star \star}$ & $0.2^{* *}$ & $0.2^{\star \star}$ & $0.2^{\star *}$ \\
\hline
\end{tabular}

**. Correlation is significant at the 0.01 level (2-tailed);

VCR30: Visual chlorosis rating at 30 days after sowing (DAS); VCR60: Visual chlorosis rating at 60 DAS; VCR90: Visual chlorosis rating at 90 DAS; SCMR30: SPAD chlorophyll meter reading at 30 DAS; SCMR60: SPAD chlorophyll meter reading at 60 DAS; SCMR90: SPAD chlorophyll meter reading at 90 DAS; NPPP: Number of pod per plant and PYPP: Pod yield per plant

QTL analysis using the BLUPs over two seasons identified a total of 11 and 12 QTLs for VCR and SCMR, respectively (Fig. 3; Table 3). Among the 11 QTLs for VCR, two, four and five QTLs were detected for VCR30, VCR60 and VCR90, respectively. Among them qVCR30_Ah04-1 on chromosome Ah04 was a major QTL for VCR30 with a PVE of 20.0\% and LOD value of 4.4. TMV 2-NLM contributed the favourable allele at this QTL for VCR. Two major QTLs ( $q$ VCR90_Ah13-1 and qVCR90_Ah12-1) on chromosome Ah13 and Ah12 were detected for VCR90 with a PVE of $34.4 \%$ and $10.3 \%$ and LOD values of 4.3 and 4.3 respectively. 
Table 3

Identification of main effect QTLs for iron deficiency chlorosis related traits among the RILs of TMV 2 and TMV 2-NLM of peanut

\begin{tabular}{|c|c|c|c|c|c|c|c|c|}
\hline Trait & Chr & $\begin{array}{l}\text { Position } \\
\text { (cM) }\end{array}$ & $\begin{array}{l}\text { Flanking } \\
\text { distance }\end{array}$ & Left marker & Right marker & LOD & PVE & $\begin{array}{l}\text { Additive } \\
\text { effect }\end{array}$ \\
\hline \multicolumn{9}{|l|}{ VCR30 } \\
\hline qVCR30_Ah04-1 & Ah04 & 9.2 & $0.0-15.4$ & AhTE0087 & TC11H06 & 4.4 & 20.0 & -0.007 \\
\hline qVCR30_Ah16-1 & Ah16 & 2.0 & $0.0-7.1$ & AhTE0242 & AhTE0060 & 5.7 & 6.9 & 0.013 \\
\hline \multicolumn{9}{|l|}{ VCR60 } \\
\hline qVCR60_Ah03-1 & Ah03 & 130.2 & $\begin{array}{l}123.2- \\
133.8\end{array}$ & AhTE1158 & AhTE0249 & 3.1 & 6.5 & 0.042 \\
\hline qVCR60_Ah03-2 & Ah03 & 135.8 & $\begin{array}{l}133.8- \\
141.4\end{array}$ & AhTE0249 & AhTE0164 & 4.3 & 5.7 & 0.039 \\
\hline qVCR60_Ah09-1 & Ah09 & 111.1 & $\begin{array}{l}111.1- \\
112.7\end{array}$ & AhTE0303 & Ah09_117710533 & 3.6 & 3.4 & -0.030 \\
\hline qVCR60_Ah14-1 & Ah14 & 153.8 & $\begin{array}{l}151.8- \\
154.9\end{array}$ & Ah14_54578739 & Ah14_32873753 & 4.5 & 5.6 & -0.043 \\
\hline \multicolumn{9}{|l|}{ VCR90 } \\
\hline qVCR90_Ah03-1 & Ah03 & 136.8 & $\begin{array}{l}133.8- \\
141.4\end{array}$ & AhTE0249 & AhTE0164 & 5.0 & 8.4 & 0.120 \\
\hline qVCR90_Ah12-1 & Ah12 & 19.7 & $5.7-30.2$ & Ah02_100365825 & Ah12_108904988 & 4.1 & 10.3 & 0.003 \\
\hline qVCR90_Ah13-1 & Ah13 & 163.3 & $\begin{array}{l}159.3- \\
178.3\end{array}$ & Ah13_80163117 & Ah13_50074616 & 4.3 & 34.4 & 0.005 \\
\hline qVCR90_Ah18-1 & Ah18 & 27.4 & $\begin{array}{l}27.4- \\
30.8\end{array}$ & AhTE0556 & Ah18_3399929 & 4.0 & 3.8 & 0.082 \\
\hline qVCR90_Ah18-2 & Ah18 & 50.8 & $\begin{array}{l}49.8- \\
53.9\end{array}$ & AhTE0517 & AhTE0143 & 4.4 & 7.5 & 0.117 \\
\hline \multicolumn{9}{|l|}{ SCMR30 } \\
\hline$\underset{1}{\text { qSCMR30_Ah09- }}$ & Ah09 & 111.1 & $\begin{array}{l}111.1- \\
112.7\end{array}$ & AhTE0303 & Ah09_117710533 & 4.4 & 4.1 & 0.314 \\
\hline$\underset{1}{q S C M R 30 \_A h 11-}$ & Ah11 & 67.3 & $\begin{array}{l}67.3- \\
69.8\end{array}$ & AhTE0212 & AhTE0129 & 4.8 & 4.6 & -0.328 \\
\hline$\underset{1}{q S C M R 30 \_A h 13-}$ & Ah13 & 161.3 & $\begin{array}{l}159.3- \\
178.3\end{array}$ & Ah13_80163117 & Ah13_50074616 & 5.1 & 11.5 & -0.461 \\
\hline$\underset{1}{q S C M R 30 \_A h 14-}$ & Ah14 & 24.1 & $\begin{array}{l}17.1- \\
24.2\end{array}$ & AhTE0050 & AhTE0107 & 5.0 & 5.1 & 0.357 \\
\hline $\begin{array}{l}\text { qSCMR30_Ah14- } \\
\text { - }\end{array}$ & Ah14 & 32.5 & $\begin{array}{l}31.4- \\
36.2\end{array}$ & Ah14_138037990 & AhTE0001 & 4.1 & 7.3 & 0.415 \\
\hline$\underset{1}{q S C M R 30 \_A h 15-}$ & Ah15 & 32.3 & $\begin{array}{l}32.3- \\
32.4\end{array}$ & PM36 & Ah15_142386409 & 3.6 & 3.8 & -0.330 \\
\hline \multicolumn{9}{|c|}{ Chr: Chromosome; LOD: Logarithm of odds; PVE: Phenotypic variance explained; } \\
\hline \multicolumn{9}{|c|}{$\begin{array}{l}\text { VCR30: Visual chlorosis rating at } 30 \text { days after sowing (DAS); VCR60: Visual chlorosis rating at } 60 \text { DAS; VCR90: Visual } \\
\text { chlorosis rating at } 90 \text { DAS; SCMR30: SPAD chlorophyll meter reading at } 30 \text { DAS; SCMR60: SPAD chlorophyll meter } \\
\text { reading at } 60 \text { DAS and SCMR90: SPAD chlorophyll meter reading at 90 DAS }\end{array}$} \\
\hline
\end{tabular}




\begin{tabular}{|c|c|c|c|c|c|c|c|c|}
\hline Trait & Chr & $\begin{array}{l}\text { Position } \\
\text { (cM) }\end{array}$ & $\begin{array}{l}\text { Flanking } \\
\text { distance }\end{array}$ & Left marker & Right marker & LOD & PVE & $\begin{array}{l}\text { Additive } \\
\text { effect }\end{array}$ \\
\hline \multicolumn{9}{|l|}{ SCMR60 } \\
\hline$\underset{1}{q S C M R 60 \_A h 12-}$ & Ah12 & 129.5 & $\begin{array}{l}129.5- \\
140.9\end{array}$ & Ah12_1893158 & Ah12_12348612 & 4.0 & 3.7 & 0.526 \\
\hline$\underset{1}{q S C M R 60 \_A h 18-}$ & Ah18 & 27.4 & $\begin{array}{l}27.4- \\
30.8\end{array}$ & AhTE0556 & Ah18_3399929 & 3.6 & 3.3 & 0.252 \\
\hline \multicolumn{9}{|l|}{ SCMR90 } \\
\hline$\underset{1}{q S C M R 90 \_A h 12-}$ & Ah12 & 21.2 & $5.7-30.2$ & Ah02_100365825 & Ah12_108904988 & 3.8 & 11.7 & -0.251 \\
\hline $\begin{array}{l}\text { qSCMR90_Ah12- } \\
2\end{array}$ & Ah12 & 27.7 & $5.7-30.2$ & Ah02_100365825 & Ah12_108904988 & 4.2 & 5.6 & -0.834 \\
\hline$\underset{1}{q S C M R 90 \_A h 18-}$ & Ah18 & 49.8 & $\begin{array}{l}49.8- \\
53.9\end{array}$ & AhTE0517 & AhTE0143 & 3.6 & 3.3 & 0.582 \\
\hline$\underset{1}{\text { qSCMR90_Ah19- }}$ & Ah19 & 65.2 & $\begin{array}{l}65.2- \\
67.1\end{array}$ & Ah19_155127338 & Ah19_155127364 & 3.8 & 3.5 & 0.659 \\
\hline \multicolumn{9}{|c|}{ Chr: Chromosome; LOD: Logarithm of odds; PVE: Phenotypic variance explained; } \\
\hline \multicolumn{9}{|c|}{$\begin{array}{l}\text { VCR30: Visual chlorosis rating at } 30 \text { days after sowing (DAS); VCR60: Visual chlorosis rating at } 60 \text { DAS; VCR90: Visual } \\
\text { chlorosis rating at } 90 \text { DAS; SCMR30: SPAD chlorophyll meter reading at } 30 \text { DAS; SCMR60: SPAD chlorophyll meter } \\
\text { reading at } 60 \text { DAS and SCMR90: SPAD chlorophyll meter reading at } 90 \text { DAS }\end{array}$} \\
\hline
\end{tabular}

Among the 12 QTLs for SCMR, six, two and four QTLs were detected for SCMR30, SCMR60 and SCMR90, respectively

(Fig. 3; Table 3). Among them, qSCMR30_Ah13-1 on chromosome Ah13 was a major QTL for SCMR30 with a PVE of 11.5\%. Likewise, qSCMR90_Ah12-1 on chromosome Ah12 was a major QTL for SCMR90 with a PVE of 11.7\%. Of the two QTLs detected for SCMR60, the highest PVE of 3.7\% was observed for qSCMR60_Ah12-1.

In total, two QTL clusters were identified for VCR and SCMR. Cluster 1 of $24.5 \mathrm{cM}$ (5.7-30.2 cM) flanked by Ah02_100365825 and Ah12_108904988 on Ah12 chromosome was common for VCR90 and SCMR90. It showed the maximum PVE of $10.3 \%$ and $11.7 \%$ for VCR90 and SCMR90, respectively. Cluster 2 of $19 \mathrm{cM}$ (159.3-178.3 cM) region flanked by Ah13_80163117 and Ah13_50074616 on chromosome Ah13 controlled VCR90 and SCMR30 with the PVE of $34.4 \%$ and $11.5 \%$, respectively (Table 3 ).

Single marker analysis showed the significant association (LOD more than the threshold calculated using 1,000 permutations) of four markers (AhTE1144, AhTE0087, AhTE0120 and AhTE0242) for VCR30. The marker AhTE1144 showed the highest PVE of $7.7 \%$ followed by AhTE0242 (7.4\%). AhTE0087 with a PVE of $6.3 \%$ was found in the vicinity of the major QTLs qVCR30_Ah04-1 (Supplemental Table S5). An attempt was made to find epistatic QTLs for VCR and SCMR. However, genomic regions with epistatic interactions were not identified. Multiple QTL mapping could identify the same major QTLs for all the traits.

Candidate gene discovery resulted in the identification of 300 genes in cluster 1 flanked by Ah02_100365825 and Ah12_108904988 and 259 genes in cluster 2 flanked by Ah13_80163117 and Ah13_50074616 (Supplemental Tables S6 and S7). All the markers showing significant association with VCR30 through single marker analysis were non-genic. However, an SNP marker Ah14_138037990, which showed a PVE of 4.3\% with a LOD score of 3.0 for VCR30 was located in the intronic region of Arahy.QAOC1 (on chromosome Ah14) which is known to have protein binding function.

\section{Discussion}


This study reports identification of QTL for IDC-related traits like VCR and SCMR in peanut using an existing genetic map from a RIL population of TMV 2 and TMV 2-NLM and the phenotypic data collected over two seasons in iron deficient soils. With the normal distribution of VCR and SCMR, they showed significant association with the productivity traits like NPPP and PYPP though a few RILs recovered from the IDC stress at 90 DAS. VCR60 and SCMR30 together contributed for $16.2 \%$ variation at PYPP indicating the influence of VCR and SCMR on productivity. TMV 2 (Spanish type) and TMV 2NLM clearly show susceptible and tolerance responses, respectively. High PCV and GCV were recorded for VCR and SCMR among the RILs indicating the presence of substantial variability. In peanut, Spanish bunch types were found to be more tolerant to IDC than the other botanical types (Pattanashetti et al. 2018), and the pod yield was positively correlated with chlorophyll content as reflected by SCMR (Singh et al. 2018). SCMR was successfully used as an indicator of IDC tolerance (Samdur et al. 1999). High heritability and GAM indicated a high proportion of phenotypic variance which is heritable and the extent of improvement in the mean genotypic value, respectively for VCR and SCMR during the three stages (30, 60 and 90 DAS).

Though 11 QTL were identified for VCR, only three QTL; qVCR30_Ah04-1 for VCR30, and qVCR90_Ah13-1 and qVCR90_Ah12-1 for VCR90 were major regions with a PVE of 20.0\%, 34.4\% and $10.3 \%$, respectively. Of the 12 QTLs detected for SCMR, qSCMR30_Ah13-1 for SCMR30 and qSCMR90_Ah12-1 for SCMR90 were two major regions with PVE of $11.5 \%$ and $11.7 \%$, respectively. qVCR30_Ah04-1 was also supported by a marker (AhTE0087) (Fig. 4) identified through single marker analysis. However, none of these five major QTL showed epistatic interaction with other genomic regions.

Of the five major QTL, four were clustered in two genomic regions; a $24.5 \mathrm{cM}$ region on Ah12 chromosome with 300 predicted genes carried one QTL each for VCR90 and SCMR90, and a 19 cM region on chromosome Ah13 with 259 predicted genes carried one QTL each for VCR90 and SCMR30. The cluster 2 on Ah13 was consistent as it was also identified for VCR90 by Pattanashetti et al. (2020), while Pandey et al. (2021) identified a QTL cluster on Ah13 for VCR30, SCMR60 and SCMR90. These regions can be the targets for further fine-mapping. A marker (Ah14_138037990) identified with a PVE of $4.3 \%$ for VCR30 using single marker analysis was located in the intronic region of the gene Arahy.QAOC1, which is known to have protein binding function. Further fine-mapping and candidate gene discovery, as it has been reported in soybean (Merry et al. 2019, Assefa et al. 2020) and mungbean (Prathet et al. 2012), could be useful for genebased methods of improving peanut for IDC tolerance. Overall, this study validated the results of the previous studies in a different mapping population, and also provided more evidence for the QTL, especially for VCR and SCMR influencing tolerance to iron deficiency chlorosis in peanut.

\section{Declarations}

\section{Acknowledgements}

Authors acknowledge the support of Department of Genetics and Plant Breeding, College of Agriculture, Vijayapur for phenotyping and Institute of Agri-Biotechnology (IABT), UAS, Dharwad and Centre of Excellence in Genomics \& Systems Biology (CEG\&SB), ICRISAT, Patancheru, India for SNP genotyping/sequencing work. The financial support received from ICAR-NASF (New Delhi) is also acknowledged.

\section{Compliance with Ethical Standards}

Authors confirm disclosure of potential conflicts of interest. This research does not involve Human Participants and/or Animals and all the authors are informed and consent is obtained.

\section{References}

1. Assefa T., J. Zhang, R. Chowda-Reddy, A.N.M. Lauter, A. Singh, J.A. O’Rourke, M.A. Graham \& A.K. Singh, 2020, Deconstructing the genetic architecture of iron deficiency chlorosis in soybean using genome-wide approaches. BMC 
Plant Biol 20: 1-13.

2. Boodi I.H., S.K. Pattanashetti, B.D. Biradar, G.K. Naidu, V.P. Chimmad, A. Kanatti, V. Kumar \& M.K. Debnath, 2016, Morpho-physiological parameters associated with iron deficiency chlorosis resistance and their effect on yield and its related traits in groundnut. Journal of crop science and biotechnology 19: 177-187.

3. Ding H., L. Duan, H. Wu, R. Yang, H. Ling, W.X. Li \& F. Zhang, 2009, Regulation of AhFRO1, an Fe(III)-chelate reductase of peanut, during iron deficiency stress and intercropping with maize. Physiol Plant 136: 274-283.

4. Hake A.A., K. Shirasawa, A. Yadawad, M. Sukruth, M. Patil, S.N. Nayak, S. Lingaraju, P.V. Patil, H.L. Nadaf, M.V.C. Gowda \& R.S. Bhat, 2017, Mapping of important taxonomic and productivity traits using genic and non-genic transposable element markers in peanut (Arachis hypogaea L.). PLoS One 12: e0186113.

5. Jadhav M.P., S.S. Gangurde, A.A. Hake, A. Yadwad, S.M. Supriya, S.K. Pattanashetti, M.V.C. Gowda, K. Shirasawa, R.K. Varshney, M.K. Pandey \& R.S. Bhat, 2021, Genotyping-by-sequencing based genetic mapping identified major and consistent genomic regions for productivity and quality traits in peanut. Front Plant Sci 12: 2034, (2010.3389/fpls.2021.668020).

6. Kong J., Y. Dong, L. Xu, S. Liu \& X. Bai, 2014, Effects of foliar application of salicylic acid and nitric oxide in alleviating iron deficiency induced chlorosis of Arachis hypogaea L. Bot Stud 55: 9.

7. Merry R., K. Butenhoff, B.W. Campbell, J.M. Michno, D. Wang, J.H. Orf, A.J. Lorenz \& R.M. Stupar, 2019, Identification and fine-mapping of a soybean quantitative trait locus on chromosome 5 conferring tolerance to iron deficiency chlorosis. Plant Genome 12: 190007.

8. Pandey M.K., S.S. Gangurde, V. Sharma, S.K. Pattanashetti, G.K. Naidu, I. Faye, F. Hamidou, H. Desmae, N.A. Kane \& M. Yuan, 2021, Improved genetic map identified major QTLs for drought tolerance-and iron deficiency tolerance-related traits in groundnut. Genes 12: 37.

9. Pattanashetti S.K., 2005, Genetic analysis of mutational origin of diversity in groundnut (Arachis hypogaea L.). University of Agricultural Sciences

10. Pattanashetti S.K., G.K. Naidu, P.K. KV, O.K. Singh \& B.D. Biradar, 2018, Identification of iron deficiency chlorosis tolerant sources from mini-core collection of groundnut (Arachis hypogaea L.). Plant Genet Resour 16: 446-458.

11. Pattanashetti S.K., M.K. Pandey, G.K. Naidu, M.K. Vishwakarma, O.K. Singh, Y. Shasidhar, I.H. Boodi, B.D. Biradar, R.R. Das \& A. Rathore, 2020, Identification of quantitative trait loci associated with iron deficiency chlorosis resistance in groundnut (Arachis hypogaea). Plant Breed 139: 790-803.

12. Prasad M.V.R., S. Kaul \& H.K. Jain, 1984, Induced mutants of peanut (Arachis hypogaea L.) for canopy and pod bearing characters. Indian J Genet Plant Breed 44: 25-34.

13. Prathet P., P. Somta \& P. Srinives, 2012, Mapping QTL conferring resistance to iron deficiency chlorosis in mungbean [Vigna radiata (L.) Wilczek]. Field Crops Res 137: 230-236.

14. Römheld V. \& H. Marschner, 1986, Evidence for a specific uptake system for iron phytosiderophores in roots of grasses. Plant Physiol 80: 175-180.

15. Samdur M., R. Mathur, P. Manivel, M. Ghewande \& A. Bandyopadhyay, 1999, Evaluation of advanced breeding genotypes of groundnut for resistance to major foliar fungal diseases. Curr Sci 79: 211-214.

16. Singh A., 2001, Yield losses in groundnut due to micronutrient deficiencies in calcareous soils of India. In: Plant nutrition. Springer, p 838-839

17. Singh A. \& V. Chaudhari, 1993, Screening of groundnut germplasm collection and selection of genotypes tolerant of lime-induced iron chlorosis. J Agric Sci 121: 205-211.

18. Singh A.L., R. Nakar, V. Chaudhari, K. Chakraborty, N. Goswami, K. Kalariya, C.B. Ajay, P.V. Zala \& C.B. Patel, 2018, Physiological efficiencies in 186 peanut cultivars of various botanical groups. Indian J Exp Biol 56: 899-913. 
19. Wang J., H. Li, L. Zhang \& L. Meng, 2014, Users' manual of QTL IciMapping. The Quantitative Genetics Group, Institute of Crop Science, Chinese Academy of Agricultural Sciences (CAAS), Beijing 100081, China, and Genetic Resources Program, International Maize and Wheat Improvement Center (CIMMYT), Apdo. Postal 6-641, 06600 Mexico, D.F., Mexico.

20. Wang S., C. Basten \& Z. Zeng, 2007, Windows QTL cartographer 2.5. North Carolina State University

21. Xiong H., T. Kobayashi, Y. Kakei, T. Senoura, M. Nakazono, H. Takahashi, H. Nakanishi, H. Shen, P. Duan, X. Guo, N.K. Nishizawa \& Y. Zuo, 2012, AhNRAMP1 iron transporter is involved in iron acquisition in peanut. J Exp Bot 63: 44374446.

22. Zeng Z.B., 1994, Precision mapping of quantitative trait loci. Genetics 136: 1457-1468.

23. Zhao C., L. Jia, Y. Wang, M. Wang \& M. McGiffen Jr, 2015, Effects of different soil texture on peanut growth and development. Commun Soil Sci Plant Anal 46: 2249-2257.

24. Zheng S.J., 2010, Iron homeostasis and iron acquisition in plants: maintenance, functions and consequences. Ann Bot 105: 799-800.

\section{Figures}
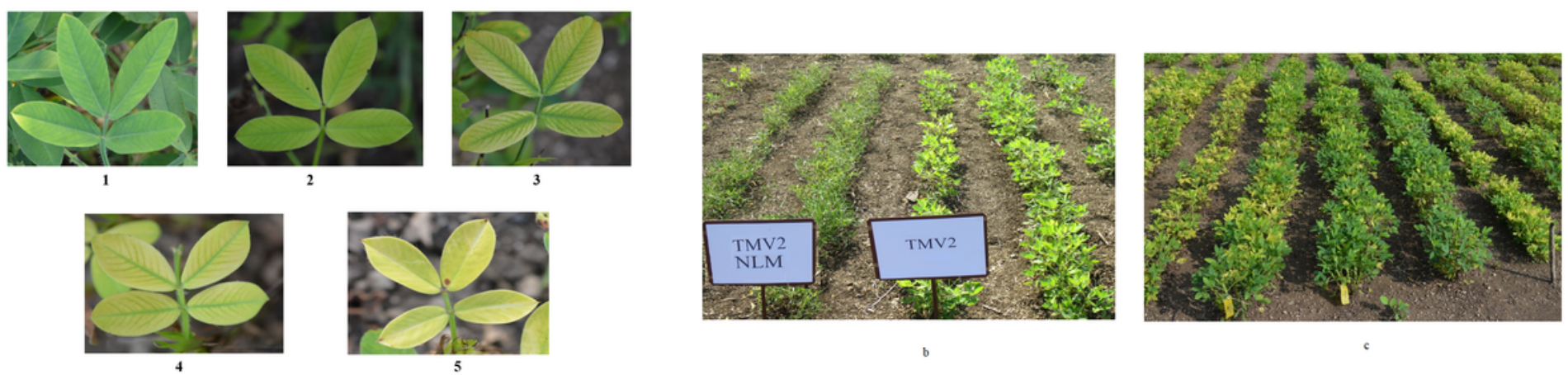

a

b

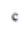

Figure 1

a Visual chlorotic rating used for visual screening for iron deficiency chlorosis in peanut

b Response of parent for iron deficiency chlorosis

c Response of the RIL population for iron deficiency chlorosis 
(a)

(b)
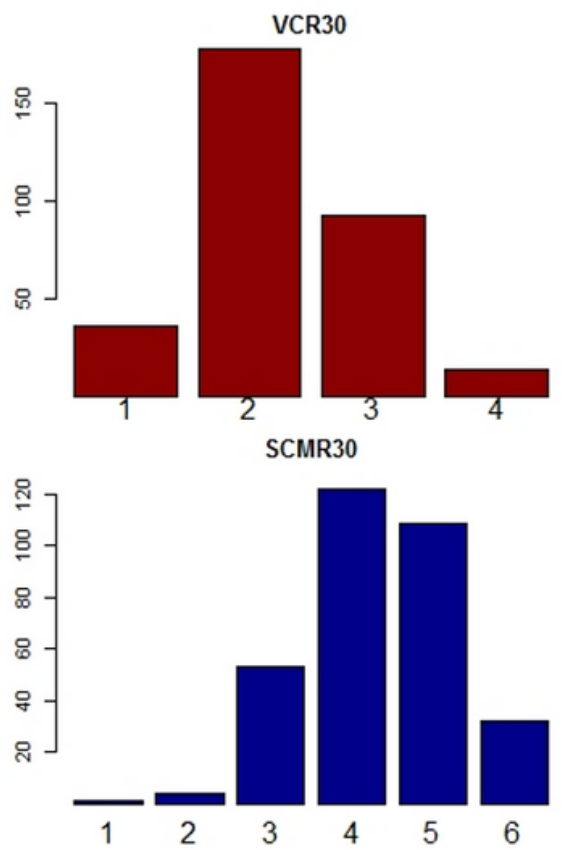

VCR60
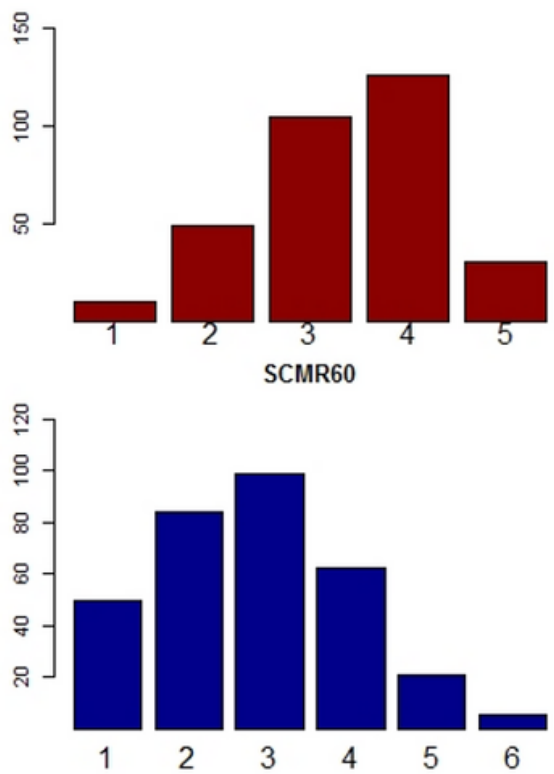

VCR90
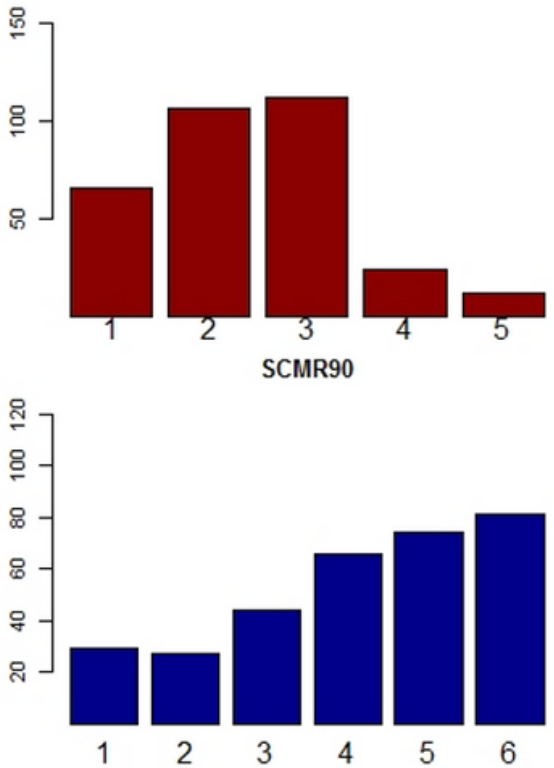

Figure 2

Frequency distribution of RILs for VCR and SCMR

(a) frequency of RILs and parents for VCR score (1 to 5) across three stages (30,60 and 90 DAS) over two years (b) frequency of RILs and parents (number) for SCMR groups (6) across three stages (30,60 and 90 DAS) over two years 


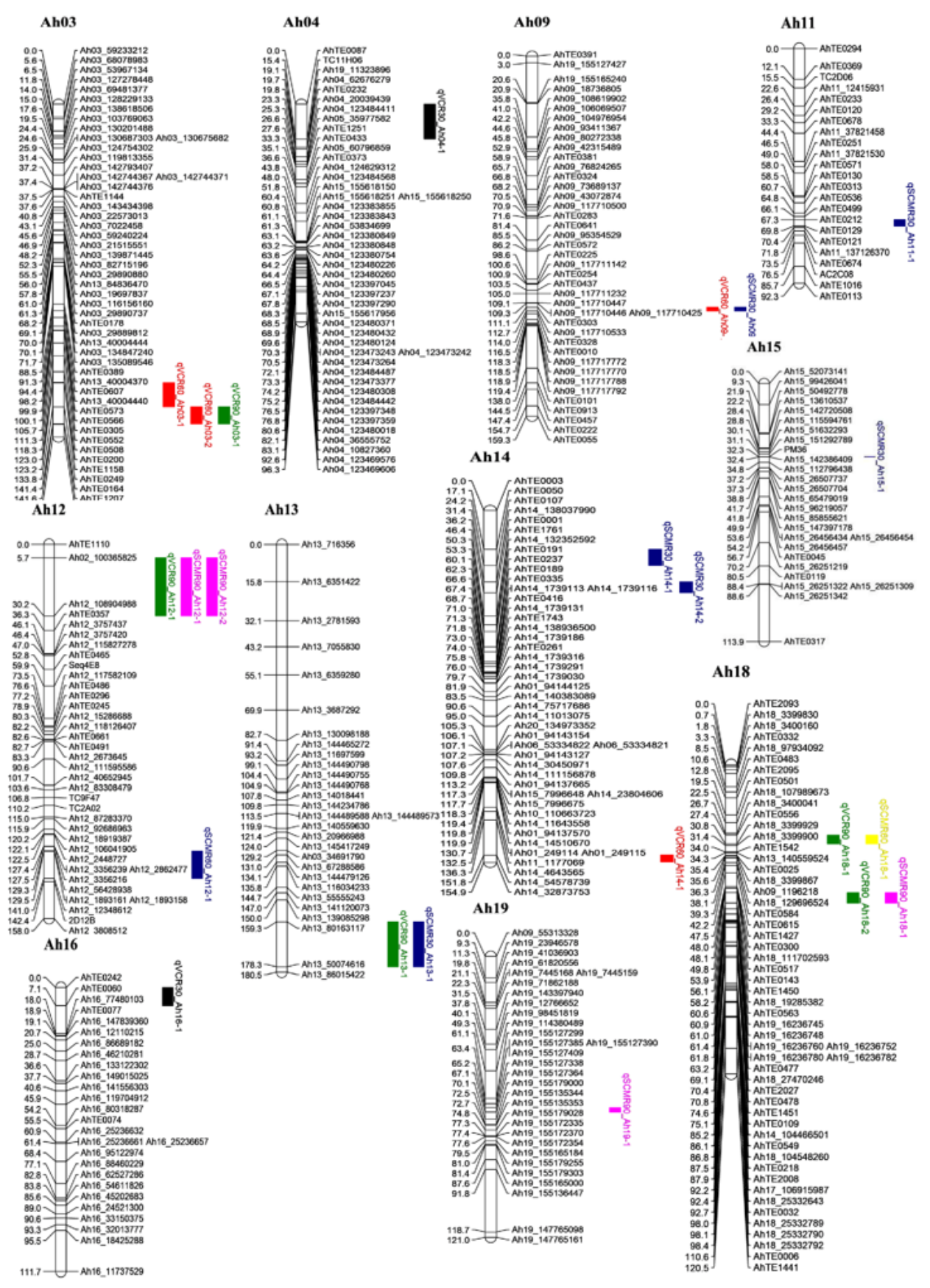

Figure 3

Genetic map showing location of QTL in different chromosome for iron deficiency chlorosis related traits among the RILs of TMV 2 and TMV 2-NLM 

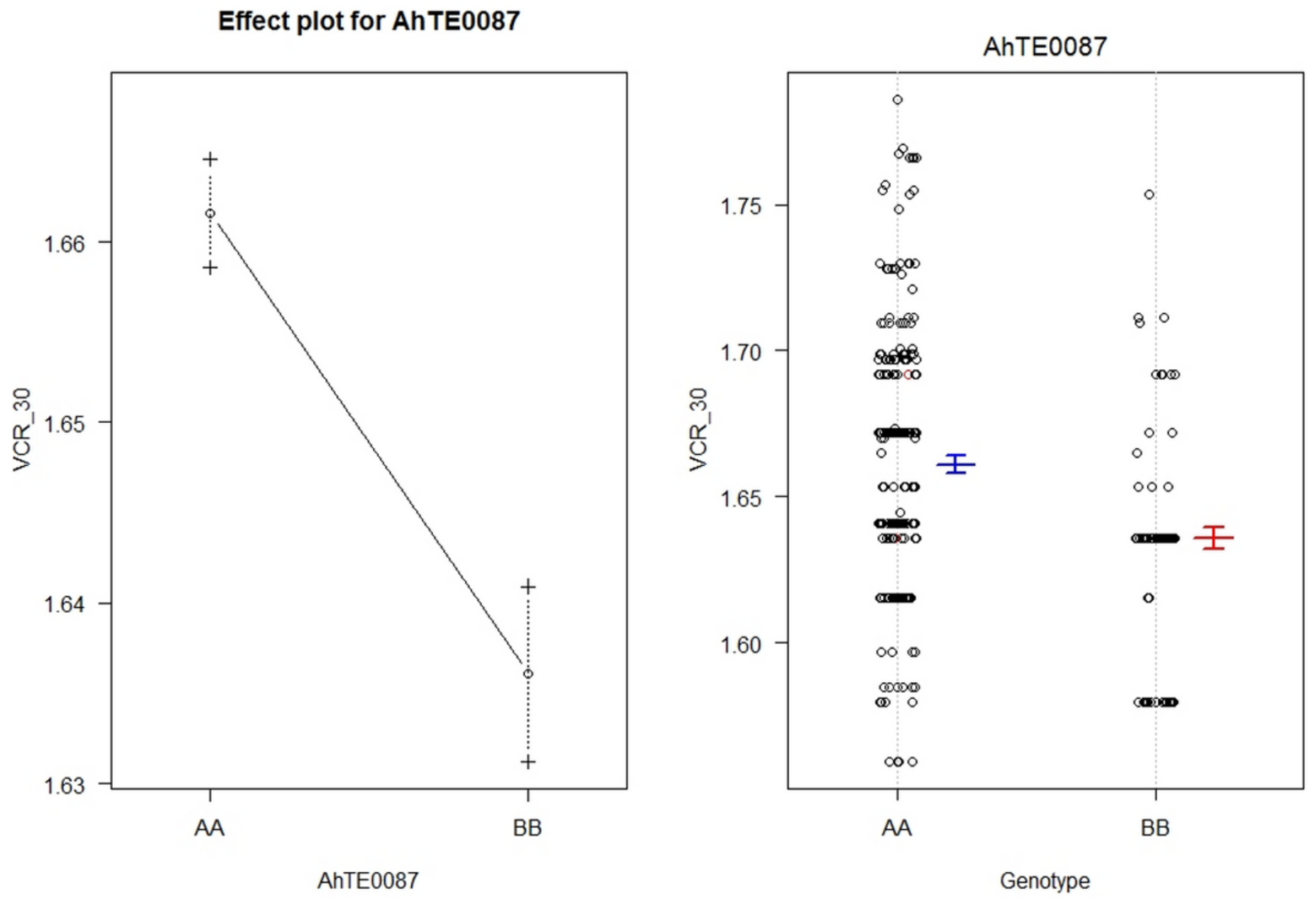

\section{Figure 4}

Mean of the genotypic classes and the additive effect for AhTE0087

\section{Supplementary Files}

This is a list of supplementary files associated with this preprint. Click to download.

- SupplementalTableS1.xlsx

- SupplementalTableS2.xIsx

- SupplementalTableS3.xlsx

- SupplementalTableS4.xlsx

- SupplementalTableS5.xlsx

- SupplementalTableS6.xlsx

- SupplementalTableS7.xlsx 